headache) and admission rates were compared at the end of therapy.

Results: The flunisolide group had significantly better PEFR at 90, 120, 150 and 180 minutes, and better $\mathrm{FEV}_{1}$ at 120, 150 and 180 minutes than did the placebo-treated group. The difference between groups increased with time. Four flunisolide-treated patients and 12 placebo-treated patients required admission $(8.5 \%$ v. $25.5 \%$; number needed to treat $[\mathrm{NNT}]=6$ ). A difference of this magnitude would be clinically significant, but given the sample size, this outcome did not achieve statistical significance. Subgroup analysis showed that placebo-treated patients whose symptom duration was greater than 24 hours had significantly lower $\mathrm{FEV}_{1}$ and higher clinical scores (including dyspnea, wheezing and accessory muscle use) at 120,150, and 180 minutes than flunisolide-treated patients (regardless of symptom duration) or placebo-treated patients with symptom duration less than 24 hours. There was no difference in adverse effects between treatment and control groups.

\section{Comments}

Study patients did not receive oral or intravenous corticosteroids, so we cannot determine whether inhaled corticosteroids have an additive effect to intravenous or oral corticosteroids. Study patients received a particularly high dose of flunisolide ( $6 \mathrm{mg} / \mathrm{h}$ over 3 hours; total, $18 \mathrm{mg}$ ). The usual dose recommended for maintenance is 1 to $2 \mathrm{mg} / \mathrm{d}$. The cost of a flunisolide inhaler containing $25 \mathrm{mg}(100 \times$
$250 \mu \mathrm{g}$ ) is $\$ 15$. The cost of one 50 -mg prednisone tablet is $\$ 0.07$. Other studies have found conflicting results: Sung and colleagues ${ }^{1}$ found a trend toward improved pulmonary index score when inhaled budesonide was added to oral prednisone in acute pediatric asthma. Guttman and cowork$\mathrm{ers}^{2}$ showed no effect when inhaled beclomethasone was added to intravenous methylprednisolone in adults with acute asthma. Scarfone and colleagues ${ }^{3}$ found nebulized dexamethasone was as effective as oral prednisone in the ED treatment of moderate asthma in children.

\section{Recommendations}

Current evidence does not support the routine use of inhaled steroids in all patients who present with acute asthma to the ED. However, inhaled steroid use in the ED may be beneficial in some patients. A systematic review or large, randomized controlled trial is warranted.

\section{References}

1. Sung L, Osmond MH, Klassen TP. Randomized, controlled trial of inhaled budesonide as an adjunct to oral prednisone in acute asthma. Acad Emerg Med 1998;5:209-13.

2. Guttman A, Afilalo M, Colacone A, Kreisman H, Dankoff J. The effects of combined intravenous and inhaled steroids (beclomethasone dipropionate) for the emergency treatment of acute asthma. Acad Emerg Med 1997;4:100-6.

3. Scarfone RJ, Loiselle JM, Wiley JF 2d, Decker JM, Henretig FM, Joffe MD. Nebulized dexamethasone versus oral prednisone in the emergency treatment of asthmatic children. Ann Emerg Med 1995;26:480-6.

\title{
SElected Articles
}

\section{Chest pain observation units for patients with unstable angina}

\section{Article chosen}

Farkouh ME, Smars PA, Reeder GS, Zinsmeister AR, Evans RW, Meloy TD, et al. A clinical trial of a chest-pain observation unit for patients with unstable angina. Chest Pain Evaluation in the Emergency Room (CHEER) Investigators. N Engl J Med 1998;339(26):1882-8.

\section{Objective}

To determine whether a chest pain observation unit (CPU) is a safe, effective and economical way to manage patients

\section{Reviewers}

Erica Rabin, MD; Michael Bullard, MD

University of Alberta, Edmonton, Alta.

Date studied: Jan. 16, 1999

This article has been peer reviewed.

with unstable angina who are at intermediate risk for shortterm cardiovascular events. 


\section{Background}

Approximately 5 million Americans undergo evaluation in the emergency department (ED) for chest pain annually, costing more than US $\$ 6$ billion. A majority are admitted for an average of 1.9 days at a mean charge of US\$4135. Almost half of admitted patients having suspected unstable angina receive a noncardiac diagnosis. ED CPUs have been proposed as a cost-effective approach to treating these lowrisk patients. According to AHCPR data, ${ }^{1} 54 \%$ of all patients with unstable angina are at intermediate risk (approximately 7\%) for a short-term death or myocardial infarction (MI).

\section{Population studied}

Of 2517 patients who presented with acute chest pain to the Mayo Clinic between November 1995 and March 1997, 424 were enrolled. Inclusion criteria were: more than 20 minutes of angina while at rest; new-onset exertional angina (Canadian Cardiovascular Society [CCS] class III or higher ${ }^{1}$ ); or variant or post-MI angina. Exclusion criteria were: ST-segment elevation, obvious noncardiac pain; a coexisting condition requiring admission; low- or high-risk unstable angina as defined by AHCPR criteria; ${ }^{1}$ or ST depression in several leads (not part of the CCS classification). Patients were also excluded if they were "out-of-area" residents, if they used English as a second language, and based on emergency physician clinical judgement. A total of 81 patients $(3.2 \%)$ refused to participate. The mean age of study subjects was 58 years and $56 \%$ were men.

\section{Study design and validity}

Patients were randomly assigned to either monitored bed admission $(n=212)$ or CPU observation $(n=212)$. Patients in the CPU group were monitored, received $325 \mathrm{mg}$ of acetylsalicylic acid, had creatine kinase (CK) and CK-MB isoenzyme levels checked at 0,2 and 4 hours, and were observed for a minimum of 6 hours. Those who had recurrent angina, ventricular dysrhythmia or elevated cardiac enzymes were admitted, and those who had an uncomplicated CPU observation period underwent provocative testing for cardiac ischemia (usually a treadmill test) before discharge.

Patients randomized to the "monitored bed admission" group received "usual" cardiology service care, which was not well described and apparently not standardized. Although treatment crossover occurred for those in the CPU group who were subsequently admitted, all calculations were made on an intention-to-treat basis. Only 2 patients, 1 from each group, were lost to follow-up during the first 6 months.

\section{Outcomes measured}

Primary outcomes included nonfatal MI, death, acute congestive heart failure (CHF), cerebrovascular accident (CVA), and out-of-hospital cardiac arrest. Secondary outcomes included additional visits to the ED for chest pain, the need for specialized cardiac diagnostic tests or procedures, and hospitalization for cardiac care within the next 6 months. Post hoc cost analyses were performed using resource-based relative value units from published sources.

\section{Results}

Ninety-seven (46\%) of 212 patients assigned to the CPU had an uncomplicated stay and negative provocative tests, allowing them to be discharged home. This led to an absolute $45.8 \%$ lower admission rate compared with those in the routine admission group. Events occurring within 30 days included 14 versus 5 nonfatal MIs, 3 versus 1 episodes of $\mathrm{CHF}$ and 0 versus 2 deaths in the non-CPU and CPU groups respectively. During the 6-month follow-up period, there were 23 primary cardiac events in the hospitalized group and 18 in the CPU group. None of the cardiac events in the CPU group occurred in patients who were discharged after a negative provocative study.

After adjusting for age, sex, previous MI, and the history of a revascularization procedure, the authors found no significant difference between groups for the risk of a primary outcome event at 30 days or at 6 months. Estimated costs were $61 \%$ higher for the hospital admission group.

\section{Study conclusion}

An ED CPU is a safe, effective and economical means of providing appropriate care to patients with unstable angina at intermediate risk for cardiovascular events.

\section{Comments}

Health care funding cutbacks, congested EDs and a growing elderly population make it critical that we find a safe and cost-effective way of dealing with ischemic chest pain patients in the ED. CPUs or chest pain programs can provide a standardized approach based on risk stratification ${ }^{1}$ and the potential for safe discharge planning. Such an approach might reduce costs in some settings.

Proving safety, however, is difficult. Farkouh and colleagues study the CPU as a therapeutic intervention, comparing it to a control intervention (CCU admission). They conclude that primary event rates were not significantly different between the 2 groups and state that their study had $80 \%$ power to detect a difference of $8 \%$ to $10 \%$ in adverse event rates. Unfortunately, this is inadequate power; a study 
of this size is likely to miss a clinically important 5\% difference in death rates between groups.

Another concern about this study is that the patient sample appeared to be of low rather than intermediate risk. This may have occurred because the protocol excluded patients with ST-segment depression in several leads and allowed emergency physicians to subjectively exclude patients with whom they were uncomfortable. In addition, although outcomes were statistically similar, the fact that there were 14 early MIs (6.6\%) in the non-CPU group versus 5 (2.4\%) in the CPU group may indicate that patients in the CPU group were healthier to begin with.

In this study and another by Gomez and associates, ${ }^{2}$ no cardiac events occurred among those CPU patients who were discharged after a negative stress test. Unfortunately both studies were underpowered and safety cannot be concluded.

Several studies cite cost efficacy as a major benefit of CPUs. Because these studies compare CPU costs only to costs for admitted patients, not to similar patients who were discharged from the ED with lesser work-ups, the true cost advantage of CPUs remains uncertain. Further, cost analysis may be inappropriate until the clinical safety of CPUs is better proven.

Published literature on CPUs suggests that discharging low- and intermediate-risk patients from the ED after a stable observation period and negative provocative test is a safe practice; however, before this can be recognized as the standard of care, a larger multicentre study or sufficiently powered systematic review should be undertaken.

\section{References}

1. Braunwald E, Jones RH, Mark DB, Brown J, Brown L, Cheitlin $\mathrm{MD}$, et al. Diagnosing and managing unstable angina. Quick Reference Guide for Clinicians. No 10. Rockville (MD): National Heart, Lung, and Blood Institute; 1994. AHCPR publ. no. 94-0602.

2. Gomez MA, Anderson JL, Karagounis LA, Muhlstein JB, Mooers FB. An emergency department-based protocol for rapidly ruling out myocardial ischemia reduces hospital time and expense: results of a randomized study (ROMIO). J Am Coll Cardiol 1996;28:25-33.

\section{Editor's note:}

This study suggests that some patients with "rule-out" unstable angina can be safely discharged after an abbreviated CPU work-up. Unfortunately, because of the nature of the enrolment criteria, it's unclear which patients these are. The study also shows that if you normally admit low-risk patients to a CCU (unlikely in Canada), you can reduce costs by not doing so. The study doesn't address what will happen if we widely introduce CPUs and, by protocol, subject patients (who previously would have been discharged from the ED) to intensive diagnostic algorithms. It is likely that this could lead to MORE investigations on more patients ("build it and they will come"), generating more costs, without clear evidence of improved patient outcomes.[G.I.]

\section{Our evidence-based future}

\section{Section Editors' notes:}

Evidence-based medicine (EBM) has a growing role in health care. Britain's National Health Service has mandated the development of a wide variety of evidence-based care plans, which they intend to introduce nationwide on a compulsory basis. Our own federal Health Minister is already discussing the importance of valid health outcomes research, so whether we believe in the concept or not, it is likely that the EBM wave will wash over Canada in the near future. To ensure that EBM is not abused, it is incumbent upon all of us to understand the process, its strengths, and its limitations.

We hope that many of you will submit CATs and evidence-based reviews for us to evaluate and choose from. If we receive more quality submissions than we can publish, we will arrange publication on the CAEP Web site. Please send us your reviews, your opinions, and any other suggestions you might have for innovations or additions to this section of CJEM. [D.R., M.B.] 